\title{
Musical calling as a behavior ancestral to all modern primates
}

David M. Schruth, Ph.D.

University of Washington

\begin{abstract}
Music is a singular behavior humans exhibit via the creation of patterned sounds organized, for example, along rhythmic and melodic dimensions. Music-like behavior, however, also appears in many other animals including non-human primates. Calls with western musical attributes are primarily ascribed to gibbons, tarsiers, indri, and potentially also marmosets, tamarins, and titi monkeys. A recent survey of primates, using a continuous measure of acoustic musicality, suggests that many other species - including galagos, several lemurs, and some Old World monkeys—also exhibit calls with music-like qualities. Based on this ubiquity and a recent arboreal-origins theory for musical behavior, I hypothesized that the ancestor of all living primates was likewise also somewhat musical. Ancestral character estimation was used to reconstruct ancestral phylogenetic states using scored spectrographic depictions of vocalizations of extant primates ( $n=58$ species) to determine if musical calling is the initial primate condition. The results suggest that nearly all primate families have an ancestor with at least one moderately music-like call— two or more reappearing syllables—-with the notable exception of Cercopithecinae. The Papionini subset of these cheek-pouch monkeys_-which includes geladas, baboons, macaques, and mandrills—likely experienced a multi-million year diminution in musical behavior, perhaps in conjunction with their recent adaptations for quadrupedal terrestriality. These findings largely indicate that exhibition of at least one music-like call is a normal, and likely quite ancient, constituent of primate vocalization behavior.
\end{abstract}

Keywords: acoustics, singing, ace, transposition, repetition, melisma

License: Attribution-NoDerivatives 4.0 International (CC BY-ND 4.0)

This work, its predecessors, and any ideas therein may be shared freely, assuming that academic attribution standards, via citation, are honored.

DOI: $\underline{10.31234 / \text { osf.io/mkze8 }}$

First-Published: 2020/12/29

Last-Modified: 2021/03/31 


\section{Introduction}

In nearly every culture, humans exercise the curious behavior of music creation (Brown and Jordania, 2013). This behavior can often be observed as a part of ritual, in rearing of infants, in healing, or more often than not, in accompaniment to dancing (Mehr et al., 2020b). Music can bring people of a community together and perhaps also to send neighbors credible signals to garner respect of mutually enforced boundaries (Mehr et al., 2020a). But the study of the function in humans is challenging because music appears to be inextricably enshrined in our cultural backgrounds as well as wired into our individual aesthetic preferences (Schruth et al., 2019). This difficulty is related to the general methodological hurdles_-including ethical, lifespan, and bias_-of objectively studying ourselves. Thus, coming up with a culture-independent way of describing music, is as important as, if not prerequisite to, exploring originating causes (Schruth et al., 2019). This bias also surfaces for the problem of looking for music-like behavior in other animals besides humans.

Musical behavior has been well studied in a diversity of animals aside from humans including birds, whales, and non-human primates (Hauser and McDermott, 2003). We can observe particular aspects of musicality (e.g. rhythm, entrainment, pitch), however, in taxa as diverse as insects (Pollack and Hoy, 1979), seals (Ravignani, 2018), elephants (Hoeschele et al., 2015), canids (Hagen and Hammerstein, 2009), and even fish (Balebail and Sisneros, 2020). Discovering a common origin of musical behavior in these various species suffers from similar problems to that of studying music's origins in humans. There is no plain definition through which we can adhere such phenomenon to classification while also remaining amenable to the independent study of plausible evolutionary influences (Schruth et al., 2019). That is, this binary "musical” outcome variable of evolutionary processes is either too low-resolution, or too un-differentiable from originating contexts, to readily yield any major breakthroughs without adjustment.

In primates, musical species have been circumscribed to a handful of taxa including indri, gibbons, some tarsiers (Haimoff, 1986), titi (Geissmann, 2000), and possibly also marmosets and tamarins (Snowdon, 2001). A recent analysis, however, has uncovered many other species of primate (at least 10 out of 16 families) that could also be considered to have musical aspects to their calls, even after considering group size effects (Schruth et al., 2020). Many other primates, besides the four aforementioned families, emerge as having musical calls, including Galagidae, Cheirogaleidae, Pitheciidae, Lepilemuridae, Cercopithecidae, and Hominidae (Fig 1). Several leafmonkey species, sub-family Colobinae, also show indications of musical quality in some of their calls (Harris, 2006; Meyer et al., 2012). This alternative approach, in contrast to traditional binary indicators of musicality, leaves only a few non-musical families of primate such as lorisids and ayeayes. 
More exhaustive investigation into the full range of vocal capabilities of a broad sampling of primates is facilitating the revelation a much higher prevalence of primate species with ostensibly musical calls. This allows for other, more generic ecological forces, such as arboreality, to explain the evolution of musical calling in primates. The acoustic adaptation hypothesis suggests that animals will develop calls that improve propagation through particular habitats (Morton, 1975). Features such as low frequencies and syllables transposed across large intervals are thought to help overcome attenuation of a signal through such obstructive substrate (Ey and Fischer, 2009). Similarly, the acoustic niche hypothesis posits that species will evolve calls which fill certain unoccupied bandwidths within the larger socio-environmental sound-scape (Krause, 1993). Strictly social explanations, in contrast, may instead extol conjoinment effects as the primary evolutionary explication of musical calling, perhaps neglecting a concern that the social phenomenon is not entirely inseparable from the acoustic one.

The fortuitous discovery of music-like behavior by primatologists over the years may have subconsciously drawn upon notational conventions of, invariably culturally western, music instruction. This preconception may be related to biomusicolgists' understandable confounding of musical behavior (e.g. temporally entrainable patterns) with socially simultaneous performance such as duetting (Ravignani, 2014). As an unfortunate side-effect of this consolidation, however, only monogamous species such as Tarisus spectrum have typically been considered musical (Haimoff, 1986) where other non-monogamous or non-duetting members of the same genus tend to get excluded. But this conflation of multiple singers with musical behavior may be an artifact of the western musical tradition of transcribing notes to paper to coordinate such performance. A context independent musicality measure, however, possibly helps to overcome this group-coordination logistics bias (Schruth et al., 2019). Thus, progress beyond the traditional, but low-resolution, binary musicality indicator, to a spectrally as well as temporally accommodating measure-one amenable to detecting environmental as well as social influences-is incumbent.

I collected the simplest possible structural features of acoustic musicality and analyzed them using principal components analysis to inform a feature reduction into such a univariate measure. This previously developed index of acoustic musicality, ARDI, was used to reconstruct the ancestral states of primate musicality from the species level up to the infra-order level. I find not only that nearly every primate family contains species with calls that have music-like qualities, but that the ancestor of all living primates likely exhibits a moderate degree of musicality in at least one call. In contrast to coarser attention to the average from each species, my search for the maximum musicality within a species' vocal repertoires inverts the commonly held understanding of musical prevalence in primates from just some species to most species. 


\section{Methods}

Spectrograms from all primate families were sampled ( $n=58$ species) using systematic searches of the academic literature via Web of Science Citation Index (Garfield, 1970) and Google scholar (Acharya and Verstak, 2004). Unique syllables, repetition, and transposition (selected via a PCA reduction procedure) were assessed by five scorers blind to both call names and caller taxa. Syllable count, the foundation of our metric, was tallied by counting unique melismatic shapes, but was then also down-weighted by multiplication to its reappearance probability — calculated via the addition of its within-call transposition and repetition probabilities. This combination ensures that all calls have non-zero representation of these contrasting acoustic aspects-helping to appropriate a fundamental counterbalance between musical variety and redundancy. Details of this scoring protocol (Schruth, 2020), the PCA reduction procedure, and ARDI index construction is detailed elsewhere (Schruth et al., 2019). Raw spectrograms, source sampling information, training materials, musical feature scores, and PCA code are available at OSF online ${ }^{1}$.

Another index of song complexity [SCI]—a euclidean-based measure emphasizing distinct unit counts, unit repetition, and call length (Sawant et al., 2019), calculated via an approximation summarized elsewhere ${ }^{2}$ — was subsequently used in an auxiliary ancestral reconstruction to independently corroborate the ARDI-based musicality reconstruction. Values of SCI were logarithmically rescaled to help normalize the distribution — towards an end goal of plotting a more homogeneous value gradient and to therefore more readily enable comparisons with ARDI values. Checks were run (Freckleton, 2009) on both sets of complexity values to ensure conformation to normality assumptions.

I used ancestral character estimation [ACE] to triangulate the (continuous) values of call complexity of the internal and root nodes of the primate phylogenetic tree-specifically the restricted maximum likelihood [REML] method from the ape R package (Paradis et al., 2004), which estimates nodes based on transformed tree topologies. The selection of kappa=1.8 was based on the average of ML estimates from several other phylogenetic regression (Orme et al., 2012) experiments performed in other recent studies on the same primate tree for the same outcome variable (Schruth, et.al. 2020). I also assumed a scaled Brownian motion binary parameter for the model, though multiple combinations were tested. Additionally, phylogenetic signal was assessed via calculations of both Pagel's $\lambda$ (Pagel, 1999) and Blomberg's K (Blomberg et al., 2003) via the phylosig function in the phytools (v. 0.2-1) R package (Revell, 2012). For all estimates, I used a composite phylogenetic tree calculated from both nuclear and mitochondrial genomes (Springer et al., 2012).

1https://osf.io/bvsfz/ "structural acoustic features of human musicality scored on primates" project 2https://osf.io/x6ch8 SCI approximation simulation using only call length, syllables, and repetition 


\section{Results}

Phylogenetic signal, as measured by Pagel's $\lambda$, was significantly non-zero for ARDI (Table 1) indicative of trait evolution corresponding to brownian motion. According to ACE analysis, nearly every primate family had a parent node with a call that was somewhat music-like (Figs 1 and 2) with ARDI scores $(\mu=2.5)$ mostly above a threshold of two reappearing syllables for at least one call. Hylobatidae and Tarsiidae had, by far, the highest average values around an ARDI of four, in confirmation, Indri excepting, of previous binary notions of musicality in primates. Other reasonably high averages occurred in Ponginae, Callicebinae, Callitrichinae, Galagidae, Pitheciidae-all well above the ARDI score threshold of two, but still less than three. Hominoidea, Colobinae, Cebinae and Lemurinae were all surprisingly just above this threshold. Other lessmusical families included Lorisidae, Daubentoniidae, and potentially the Atelidae who only had data for one species per family but also scored below this threshold. Only the family Ceropithecinae, consisting mostly of tribe Papionini, averaged decidedly below this (admittedly arbitrary) threshold of two reappearing syllables, an estimate supported by data from 13 species. Estimates of musicality using $\log (\mathrm{SCI})$ were relatively similar to those of ARDI except that it tended to highlight longer, more repetitive calls, like those of Chimpanzees.

Table 1. Phylogenetic signal via both Pagel's $\lambda$ and Blomberg's $K$ for all complexity measures

\begin{tabular}{|c|c|c|c|c|c|c|}
\hline & \multicolumn{2}{|c|}{ syllable count } & \multicolumn{2}{|c|}{ ARDI } & \multicolumn{2}{|c|}{$\log (\mathrm{SCl})$} \\
\hline & estimate & $p$-value & estimate & $p$-value & estimate & $p$-value \\
\hline Pagel's $\lambda$ & 0.42 & $(0.046)$ & 0.75 & $(0.002)$ & 7.59E-05 & $(1.000)$ \\
\hline Blomberg's $K$ & 0.36 & $(0.357)$ & 0.23 & $(0.005)$ & 0.12 & $(0.323)$ \\
\hline
\end{tabular}




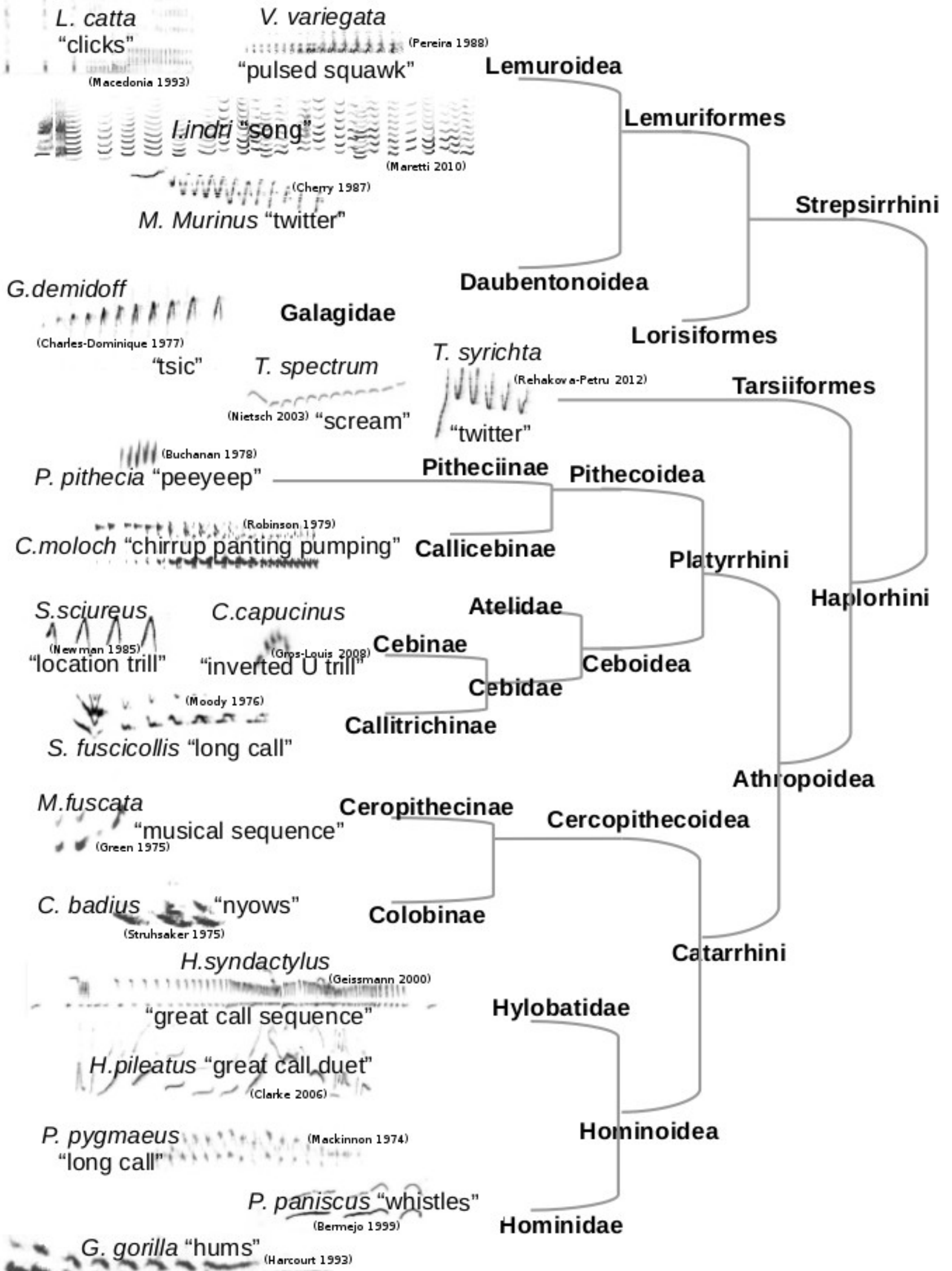

Fig 1. Various music-like calls of modern primates matched to a cladogram based on (Fleagle, 2013). This broad sampling demonstrates that most primate families have at least one species with at least one transpositionally melodic call. Also note the rhythmic patterning in the two lemur calls (top). A hyperlinked list of all spectrographic sources and examples is available at osf.io/bvsfz 

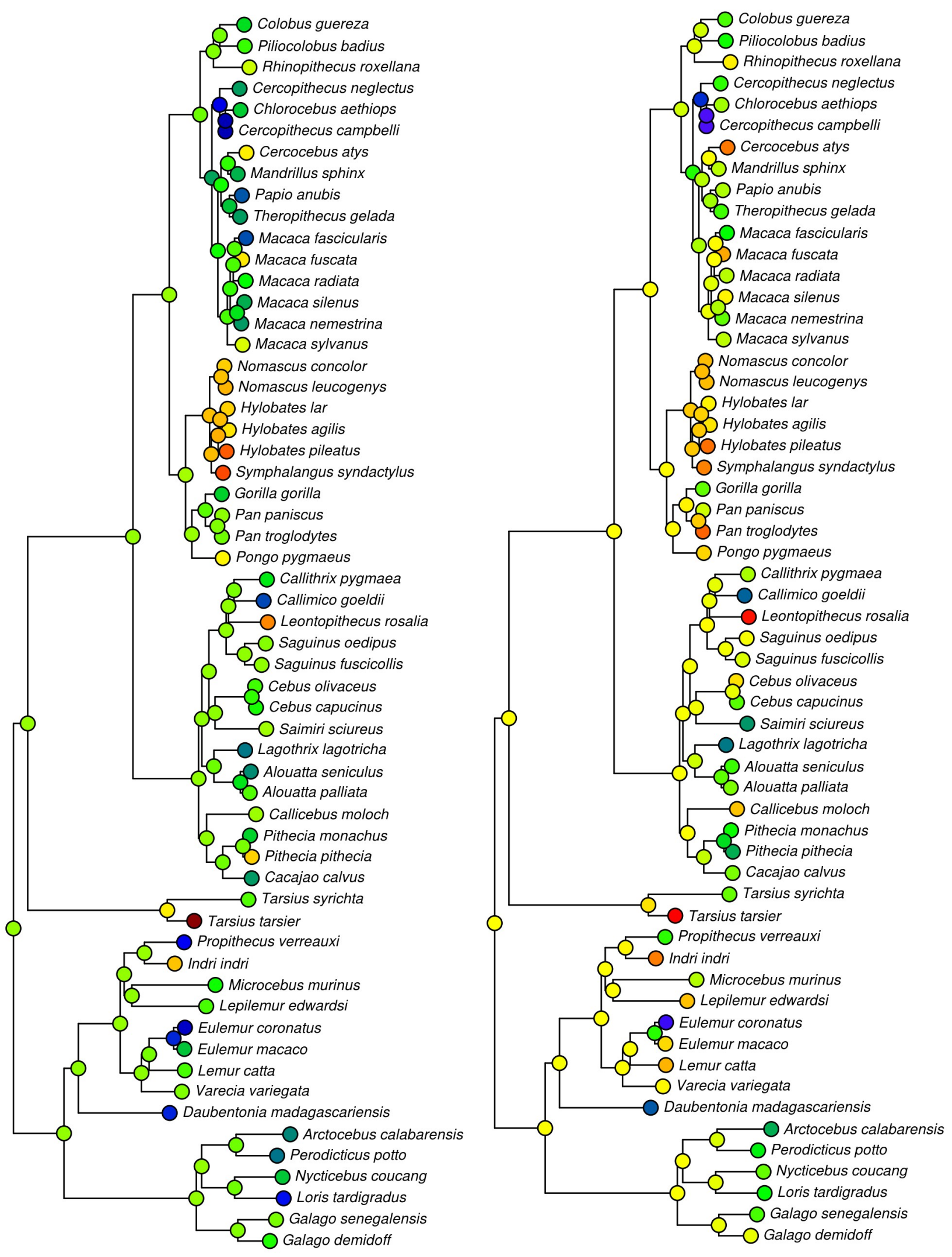

Fig 2. Phylogenetic trees with nodes calculated using both ARDI and SCI call complexity metrics. Values for each complexity metric are plotted from lower to higher values using increasingly hotter colors (blue=low, red=high). ARDI (left) tends to accentuate shorter more transpositionally musical calls like that of $P$. pithecia whereas SCI (right) highlights longer more repetitive calls such as those of C. moloch, P. troglodytes, and C. atys. 


\section{Discussion}

The unsurprising finding of this study is that the phylogenetic average of the most musical calls of a broad sample of modern primates indicates that the basal euprimate possessed a call with music-like qualities. This average ARDI value of over two reappearing syllables per call is comparable with the music-like calls of many modern primates such that of $T$. syrichta and $M$.

Murinus. Many primates previously not thought to be musical species actually do exhibit a call with music-like qualities. For example, the "tsic" call of Galagoides demidoff, the "twitter" of Microcebus murinus, the "peeyeep" of Pithecia pithecia, the "duet” of Lepilemur edwardsi, the “clicks” of Lemur catta, the “pulsed squawk” of Varecia variegata, the "long call” of Pongo pygmaeus, and the "hum" of G. gorilla could all be argued to contain musical qualities (Fig 1). Other, non- marmoset and tamarin, members of Cebidae such as squirrel monkeys (e.g. "location trill”) and capuchins (e.g. “inverted U trill”) also seem to have some notably musical calls.

This expanded relevance of music-like calling amongst primates allows for a broadened reconsideration of potential selective influences. Since the smaller subset of primates that duet-a commonly exploited clue to a species' musicality-invariably correlates with social monogamy, a sensible resolution is to also advance monogamy or social bonding as predominate evolutionary influences (Freeman, 2000; Savage et al., 2020). Admittedly, it is tempting to postulate a musicality acceleration effect corresponding to such higher levels of monogamy-compelling since the four core aforementioned musical primate families tend to be more monogamous (Schruth et al., 2019). But because we have newfound exposure to a much broader array of possibly musical species, there are other, entirely environmental possibilities that could alternatively explain the wider initial emergence. Arboreality, for example, could well explain such a broadened continuum of primate musicality since most primates are in-fact arboreal (Schruth et al., 2020). Interestingly, most of the terrestrial (tribe Papionini) monkeys, with the exception of $M$. fuscata, do not have musical calls.

Such a connection with habitat raises interesting questions about the genitors of musicality in other animals such as birds, whales, and perhaps the other more terrestrial species. It is possible that they have overcome acoustic transmission impediments by employing saliently intervalic sequences, a key feature implicated here. I have postulated elsewhere that habitat dimensionality exerts a moderate selective effect for increased patterning in both the temporal and spectral domains of musicality (Schruth, et.al. 2020). Indeed, precision landing during (e.g. gravitationally or respirationally) time-sensitive locomotor bout transitions may constitute a compelling indirect selection pressure for acoustic display. In addition to locomotion, various ways of reducing predation risks also likely factor into selection for salient vocalizing (Schruth and Jordania, 2020). While several zoologically broad origins scenarios are promising, more work is needed in 
automating evaluation and in accommodating additional, especially aquatic, animal taxa into such phylogenetic analysis.

A remaining yet unresolved curiosity is how and why musicality might be so diminished in terrestrial species. Specifically, gradual degradation of such systems of musical behavior over-time, in some primates, could help resolve the adaptive significance puzzle that exists in modern human music. If the deterioration of musicality potentially takes millions of years in terrestrial monkeys and cryptic lorises, then the same might be said of terrestrial humans who likewise are no longer arboreal and have greatly diminished olfactory capabilities. This slow deterioration could explain the widespread mystery as to music's persistence and function in our species. Perhaps there are only very few remaining environmental selection pressures maintaining the sending of such signals in most human populations. In sum, the mystery of human music could be explained by an extremely slow evolutionary decoupling of musical capabilities from selection pressures placed upon our primate ancestors. The past 60 million years of spatio-temporal ecological constraints, and the associated vocal coordination for arboreally, could have just been exceedingly slow in fully unraveling in the more recent two million years of obligate hominin terrestriality.

An important final caveat, however, is the unattended observation of an opposite trend towards greater levels of musicality, than our putatively musical ancestral primate, in both tarsiers and gibbons. The complexity, as measured here by ARDI and SCI, and other indicators of acoustic liveliness, are at higher levels than most other primates, including those estimated for ancestral taxonomic ranks. Therefore, it is still entirely possible that selection pressures in hominins, like the isolated selection pressures in these two clades, have instead increased recently_corresponding to a level of musicality which is likely to be quite high in humans, heretofore unmeasured. A unique combination of evolutionary influences, via both cultural and genetic selection processes, likely allows for a distinct rate and trajectory of musical innovation-making hominin evolutionary musicology an area ripe for indefinitely protracted study. 


\section{Reference Cited}

Acharya, A., Verstak, A., 2004. Google Scholar.

Balebail, S., Sisneros, J.A., 2020. Relationship of advertisement call parameters with phenotypic traits in "singing" male plainfin midshipman. Integrative and Comparative Biology. 60.

Blomberg, S.P., Garland, T., Ives, A.R., 2003. Testing for phylogenetic signal in comparative data: behavioral traits are more labile. Evolution. 57, 717-745.

Brown, S., Jordania, J., 2013. Universals in the world's musics. Psychology of Music. 41, 229-248.

Ey, E., Fischer, J., 2009. The "acoustic adaptation hypothesis"-a review of the evidence from birds, anurans and mammals. Bioacoustics. 19, 21-48.

Fleagle, J.G., 2013. Primate Adaptation and Evolution, 3rd ed. Academic Press, New York.

Freckleton, R.P., 2009. The seven deadly sins of comparative analysis. Journal of Evolutionary Biology. 22, 1367-1375.

Freeman, W.J., III, 2000. A neurobiological role of music in social bonding. In: The Origins of Music. MIT Press, Cambridge, Massachusetts, pp. 411-424.

Garfield, E., 1970. Citation Indexing for Studying Science. Nature. 227, 669-671.

Geissmann, T., 2000. Gibbon songs and human music. In: Wallin, N.L., Merker, B., Brown, S. (Eds.), The Origins of Music. MIT Press, Cambridge, Massachusetts, pp. 103-123.

Hagen, E.H., Hammerstein, P., 2009. Did Neanderthals and other early humans sing? Seeking the biological roots of music in the territorial advertisements of primates, lions, hyenas, and wolves. Musicae Scientiae. 13, 291-320.

Haimoff, E.H., 1986. Convergence in the duetting of monogamous Old World primates. Journal of Human Evolution. 15, 51-59.

Harris, T.R., 2006. Within- and among-male variation in roaring by black and white colobus monkeys (Colobus guereza): What does it reveal about function? Behaviour. 143, 197-218.

Hauser, M.D., McDermott, J., 2003. The evolution of the music faculty: a comparative perspective. Nature Neuroscience. 6, 663-668.

Hoeschele, M., Merchant, H., Kikuchi, Y., Hattori, Y., ten Cate, C., 2015. Searching for the origins of musicality across species. Philosophical Transactions of the Royal Society B: Biological Sciences. 370, 20140094.

Krause, B., 1993. The niche hypothesis: a virtual symphony of animal sounds, the origins of musical expression and the health of habitats. The Soundscape Newsletter.

Mehr, S.A., Krasnow, M.M., Bryant, G.A., Hagen, E.H., 2020a. Origins of music in credible signaling. Behavioral and Brain Sciences. (target article).

Mehr, S.A., Singh, M., Knox, D., Ketter, D.M., Pickens-Jones, D., Atwood, S., Lucas, C., Jacoby, N., Egner, A.A., Hopkins, E.J., Howard, R.M., Hartshorne, J.K., Jennings, M.V., Simson, J., Bainbridge, C.M., Pinker, S., O’Donnell, T.J., Krasnow, M.M., Glowacki, L., 2020b. Universality and diversity in human song. Science. 366.

Meyer, D., Hodges, J.K., Rinaldi, D., Wijaya, A., Roos, C., Hammerschmidt, K., 2012. Acoustic structure of male loud-calls support molecular phylogeny of Sumatran and Javanese leaf monkeys (genus Presbytis). BMC Evolutionary Biology. 12, 16.

Morton, E.S., 1975. Ecological sources of selection on avian sounds. The American Naturalist. 109, 17-34.

Orme, D., Freckleton, R., Thomas, G., Petzoldt, T., Fritz, S., Isaac, N., Pearse, W., 2012. Caper: comparative analyses of phylogenetics and evolution in $\mathrm{R}$.

Pagel, M., 1999. Inferring the historical patterns of biological evolution. Nature. 401, 877-884.

Paradis, E., Claude, J., Strimmer, K., 2004. APE: Analyses of Phylogenetics and Evolution in R language. Bioinformatics. 20, 289-290.

Pollack, G.S., Hoy, R.R., 1979. Temporal Pattern as a Cue for Species-Specific Calling Song Recognition in Crickets. Science. 204, 429-432. 
Ravignani, A., 2014. Chorusing, synchrony, and the evolutionary functions of rhythm. Frontiers in Psychology. 5.

Ravignani, A., 2018. Spontaneous rhythms in a harbor seal pup calls. BMC Research Notes. 11, 3.

Revell, L.J., 2012. phytools: An R package for phylogenetic comparative biology (and other things).

Savage, P.E., Loui, P., Tarr, B., Schachner, A., Glowacki, L., Mithen, S., Fitch, W.T., 2020. Music as a coevolved system for social bonding. Behavioral and Brain Sciences. 1-36.

Sawant, S., Arvind, C., Joshi, V., Robin, V.V., 2019. Defining birdsong comlexity in a species with a highly variable vocal repertoire. Indian Institute of Science Education and Research, Tirupati.

Schruth, D., Jordania, J., 2020. Singing behavior via reduced predation risk (preprint). PsyArXiv.

Schruth, D.M., Holman, D.J., 2020. Protocol for scoring animal calls on structural acoustic features prevalent in human music. Protocols.io.

Schruth, D.M., Templeton, C.N., Holman, D.J., 2019. A definition of song, using human music universals observed in primate calls. BioRxiv.

Schruth, D.M., Templeton, C.N., Holman, D.J., Smith, E.A., 2020. Evolution of primate protomusicality via locomotion. BioRxiv.

Snowdon, C.T., 2001. Social processes in communication and cognition in callitrichid monkeys: a review. Animal Cognition. 4, 247-257.

Springer, M.S., Meredith, R.W., Gatesy, J., Emerling, C.A., Park, J., Rabosky, D.L., Stadler, T., Steiner, C., Ryder, O.A., Janečka, J.E., Fisher, C.A., Murphy, W.J., 2012. Macroevolutionary Dynamics and Historical Biogeography of Primate Diversification Inferred from a Species Supermatrix. PLoS ONE. 7, e49521. 
Bermejo, M., and A. Omedes. "Preliminary Vocal Repertoire and Vocal Communication of Wild Bonobos (Pan Paniscus) at Lilungu (Democratic Republic of Congo).” Folia Primatologica 70 (1999): 328-57.

Buchanan, D. "Communication and Ecology of Pithecine Monkeys with Special Reference to Pithecia Pithecia.” Wayne State University, 1978.

Charles-Dominique, P. Ecology and Behaviour of Nocturnal Primates: Prosimians of Equatorial West Africa. London: Duckworth, 1977.

Clarke, E., U.H. Reichard, and K Zuberbuhler. "The Syntax and Meaning of Wild Gibbon Songs.” PLoS ONE 1, no. 1 (2006): e73. https://doi.org/10.1371/journal.pone.0000073.

Geissmann, T. "Duet Songs of the Siamang, Hylobates Syndactylus: I. Structure Organization.” Primate Report 56 (2000): 33-60.

Green, S. "Variation of Vocal Pattern with Social Situation in the Japanese Monkey (Macaca Fuscata): A Field Study.” In Primate Behavior, 4:1-102. New York: Academic Press, 1975.

Gros-Louis, Julie J., Susan E. Perry, Claudia Fichtel, Eva Wikberg, Hannah Gilkenson, Susan Wofsy, and Alex Fuentes. "Vocal Repertoire of Cebus Capucinus: Acoustic Structure, Context, and Usage.” International Journal of Primatology 29, no. 3 (June 2008): 641-70. https://doi.org/10.1007/s10764-008-9263-8.

Harcourt, A. H., K. J. Stewart, and M. Hauser. "Functions of Wild Gorilla Close Calls .1. Repertoire, Context, and Interspecific Comparison.” Behaviour 124 (1993): 89-122.

Macedonia, J. M. “The Vocal Repertoire of the Ringtailed Lemur (Lemur Catta).” Folia Primatologica 61 (1993): 186-217.

Mackinnon, J. "Behavior and Ecology of Wild Orangutans (Pongo-Pygmaeus).” Animal Behaviour 22 (1974): 3-.

Maretti, G., V. Sorrentino, A. Finomana, M. Gamba, and C. Giacoma. "Not Just a Pretty Song: An Overview of the Vocal Repertoire of Indri Indri.” Journal of Anthropological Sciences 88, no. 2010 (2010): 151-65.

Moody, M. I., and E. W. Menzel. "Vocalizations and Their Behavioral Contexts in Tamarin Saguinus-Fuscicollis.” Folia Primatologica 25 (1976): 73-94.

Newman, J. D. “Squirrel Monkey Communication.” In Handbook of Squirrel Monkey Research, 99-126. New York: Plenum Press, 1985.

Nietsch, A. "Outline of the Vocal Behavior of Tarsius Spectrum: Call Features, Associated Behaviors, and Biological Functions.” In Tarsiers: Past, Present, and Future, edited by E. L. Simons, Sharon L. Gursky, and P. Wright, Ch. 9. Rutgers University Press, 2003.

Pereira, M. E., M. L. Seeligson, and J. M. Macedonia. "The Behavioral Repertoire of the Blackand-White Ruffed Lemur, Varecia-Variegata-Variegata (Primates, Lemuridae).” Folia Primatologica 51 (1988): 1-32.

Řeháková-Petrů, Milada, Richard Policht, and Lubomír Peške. “Acoustic Repertoire of the Philippine Tarsier (Tarsius Syrichta Fraterculus) and Individual Variation of Long-Distance Calls.” International Journal of Zoology 2012 (2012): 1-10. https://doi.org/10.1155/2012/602401.

Robinson, J. G. "Vocal Regulation of Use of Space by Groups of Titi Monkeys Callicebus-Moloch." Behavioral Ecology and Sociobiology 5 (1979): 1-15.

Struhsaker, T. T. The Red Colobus Monkey. Chicago, IL: University of Chicago Press, 1975. 